\title{
tic\&société
}

Vol. 11, $\mathbf{N}^{\circ} 1$ | 2ème semestre 2017

L'éducation critique aux médias à l'épreuve du numérique

\section{Articuler les dimensions constitutives de l'éducation aux médias}

\section{Normand LANDRY}

\section{(2) OpenEdition \\ Journals}

Édition électronique

URL : http://journals.openedition.org/ticetsociete/2236

DOI : 10.4000/ticetsociete. 2236

\section{Éditeur}

Association ARTIC

\section{Édition imprimée}

Pagination : 7-45

\section{Référence électronique}

Normand LANDRY, «Articuler les dimensions constitutives de l'éducation aux médias », tic\&société [En ligne], Vol. 11, № 1 | 2ème semestre 2017, mis en ligne le 01 septembre 2017, consulté le 10 décembre 2020. URL : http://journals.openedition.org/ticetsociete/2236 ; DOI : https://doi.org/ 10.4000/ticetsociete.2236 
tic\&société - 11(1), 2017

\title{
Articuler les dimensions constitutives de l'éducation aux médias
}

\begin{abstract}
Normand LANDRY
Normand Landry est professeur à la TÉLUQ (Université du Québec), titulaire de la Chaire de recherche du Canada en éducation aux médias et droits humains, et chercheur au Centre de recherche interuniversitaire sur la communication, l'information et la société (CRICIS). Ses travaux se concentrent sur l'éducation aux médias, les droits de la communication, l'intimidation judiciaire, ainsi que sur la communication et les mouvements sociaux. Les recherches qu'il a menées l'ont enjoint à participer à des sommets internationaux organisés sous l'égide des Nations Unies, à intervenir auprès de groupes parlementaires et à s'investir auprès de groupes de la société civile. Normand Landry est l'auteur de plusieurs ouvrages, dont Droits et enjeux de la communication (PUQ, 2013) et de Threatening Democracy: SLAPPs and the Judicial Repression of Political Discourse (Fernwood, 2014). II est également directeur de publication (avec Anne-Sophie Letellier) du livre L'éducation aux médias à l'ère numérique : entre fondations et renouvellement (PUM, 2016). normand.landry@teluq.ca
\end{abstract}




\title{
Articuler les dimensions constitutives de l'éducation aux médias
}

Résumé : Cet article procède à l'analyse critique des écrits de langue anglaise en éducation aux médias. II en présente les dimensions constitutives et les particularités. II caractérise l'éducation aux médias en tant que champ récent et distinct, actuellement en croissance rapide. Ce champ est constitué de savoirs théoriques, de pratiques pédagogiques, de compétences recherchées et de politiques publiques. Ces éléments sont introduits et détaillés. Cet article articule également trois critiques des écrits savants. Ceux-ci entretiennent une confusion conceptuelle croissante quant à l'organisation des savoirs et des compétences qu'ambitionnent de développer les pratiques pédagogiques mobilisées en éducation aux médias. Ils peinent ensuite à articuler les dimensions constitutives du champ en cohérence les unes avec les autres. Finalement, l'analyse des politiques publiques, plus particulièrement des curriculums scolaires, est lacunaire. Les connaissances sur les contenus curriculaires et les pratiques mobilisées en milieu scolaire restent à être développées.

Mots-clés : éducation aux médias, littératie médiatique, politiques éducatives, compétences, théorie, pratiques pédagogiques.

\begin{abstract}
This article provides a critical review of the Englishlanguage literature on media education. It presents its constitutive dimensions and particularities; it further characterizes media education as a recent and distinct field growing rapidly. This field brings together theoretical knowledge, pedagogical practices, sought-after competencies and policies. These elements are introduced and described. This article also proposes three criticisms of scholarly writings. Firstly, they show a high level conceptual confusion concerning the sets of knowledge and competencies that media education tries to foster. They also struggle to articulate the constitutive dimensions of the field with relation to one another. Finally, the analysis of public policies,
\end{abstract}


Articuler les dimensions constitutives de l'éducation aux médias

particularly of school curricula, is peripheral and incomplete. Knowledge of curriculum content and school-based practices remains underdeveloped.

Keywords: media education, media literacy, educational policy, competencies, theory, pedagogical practices.

Resumen: Este artículo presenta un análisis crítico de la literatura anglosajona relativa a la educación sobre los medios, a la vez que se muestran las dimensiones y las particularidades sobre dicho particular. Caracteriza la educación sobre los medios como un área de estudio particular, reciente, y en rápido crecimiento. El campo está constituido por saberes teóricos, prácticas pedagógicas, competencias específicas y por políticas públicas. Dichos elementos son presentados y analizados. Además, este artículo considera tres críticas sobre los textos académicos al respecto. En primer lugar, existe una confusión conceptual creciente en lo relativo a la organización de los saberes y de las competencias que se esperan desarrollar con las prácticas pedagógicas utilizadas en la educación sobre los medios. En segundo lugar, los textos muestran dificultades para articular coherentemente las dimensiones constitutivas del campo. Finalmente, el análisis de políticas públicas y particularmente el de los currícula escolares, se muestra incompleto. Puede concluirse que resulta necesario desarrollar los conocimientos sobre los contenidos curriculares así como las prácticas movilizadas en el medio escolar.

Palabras clave: educación sobre los medios, políticas públicas, competencias, saberes teóricos, prácticas pedagógicas. 


\section{Normand LANDRY}

\section{Introduction $^{1}$}

Longtemps déconsidéré en milieu scolaire et négligé par les politiques publiques, le champ de l'éducation aux médias jouit désormais d'une considération plus appréciable ${ }^{2}$. Le grand public, les professionnels du secteur éducatif, les chercheurs et les décideurs politiques y portent une attention accrue.

Ce regain d'intérêt n'est guère surprenant. Les dernières décennies ont vu émerger et s'affirmer des phénomènes qui légitiment les discours sur la nécessité de préparer et d'outiller la population à faire face à un environnement médiatique complexe, changeant et multiforme. Ces phénomènes regroupent tout à la fois la médiatisation croissante des rapports sociaux, les transformations technologiques et industrielles accélérées, ainsi que l'affirmation d'enjeux sociopolitiques associés aux usages et aux pratiques médiatiques ${ }^{3}$. Ils sont étroitement associés à la diffusion à grande échelle de technologiques médiatiques numériques au sein de nos sociétés.

Dans ce contexte, le développement de l'éducation aux médias est présenté à la fois comme une réponse à des préoccupations sociales et politiques touchant à la consommation et à la production médiatique - il s'agit, en d'autres mots, d'une intervention visant la résolution de problèmes sur lesquels il a été

\footnotetext{
${ }^{1}$ La rédaction de cet article a été soutenue par le programme des Chaires de recherche du Canada et le Fonds de recherche du Québec - société et culture. Nous les remercions. Certaines sections de cet article sont tirées et adaptées de textes utilisés dans le cadre du cours "COM 3028 Éducation aux médias et littératie médiatique », offert à la TELUQ. Le lecteur est invité à s'y référer.

2 Par "champ », il est entendu un domaine spécifique d'activités constitué par des communautés agissantes qui mobilisent des savoirs et de pratiques dans les contextes institutionnels et politiques qui sont les leurs. Ces communautés sont placées en situation de coopération et de compétition avec d'autres acteurs et structurent leurs activités autour d'une identité, de pratiques et de savoirs communs.

${ }^{3}$ Parmi lesquels nous retrouvons : les activités de désinformation politique (notamment en période électorale); la généralisation d'activités non autorisées de production et de diffusion de contenus protégés par le droit d'auteur ; la propagation de discours racistes, xénophobes, haineux ou discriminatoires sur les réseaux numériques ; la conciliation de l'exercice de la liberté d'expression avec d'autres droits, valeurs et normes sociales dans les productions médiatiques.
} 
Articuler les dimensions constitutives de l'éducation aux médias

jugé opportun d'intervenir - et comme un moyen d'atteindre des objectifs sociaux, économiques et politiques ciblés. L'éducation aux médias converge avec des intérêts multiples, se présentant simultanément comme un rempart à la radicalisation et à l'insécurité, une condition à l'intégration sociale et économique (et donc, au marché de l'emploi), un prérequis à l'exercice éclairé de la citoyenneté et un levier à l'expression individuelle et collective (Bragg, 2002 ; Hoechsmann et Poyntz, 2012 ; Lee, 2010). Cette convergence d'intérêts favorise une institutionnalisation accrue de l'éducation aux médias dans les politiques publiques nationales et internationales ainsi que dans les milieux scolaires et communautaires. Elle pose également - et de manière paradoxale - le risque de l'éclatement d'un champ qui intègre des approches et des objectifs contrastés.

Ces phénomènes de croissance accélérée de l'éducation aux médias et d'un élargissement considérable des mandats qui lui sont attribués enjoignent les chercheurs à en reconsidérer les fondements, les particularités, les orientations et les contributions. Cet article répond à cet appel en effectuant une analyse critique des écrits savants de langue anglaise ${ }^{4}$. Ce faisant, il cherche à dresser un bilan et à ouvrir un dialogue entre chercheurs anglophones et francophones. II est divisé en deux sections principales, suivant une courte présentation historique. La première section balise le champ, en soulevant les fractures entre ses dimensions constitutives, la nécessité de renouvellement et les difficultés qui résultent de la prolifération des «littératies médiatiques ". La seconde section se consacre à la question de l'articulation des dimensions constitutives de l'éducation aux médias (théories, pratiques, politiques publiques) et aux lacunes constatées dans les écrits. Une insistance sur "l'articulation» interroge les liens et le degré de cohérence qu'entretiennent les dimensions constitutives entre elles. On s'interroge donc sur la capacité de pratiques pédagogiques particulières à développer des savoirs et des compétences ciblés, et au degré d'adéquation entretenu entre ces savoirs et ces compétences et les objectifs

\footnotetext{
${ }^{4}$ Cet article cite également quelques auteurs francophones qui utilisent eux-mêmes la documentation de langue anglaise dans le cadre de leurs travaux.
} 


\section{Normand LANDRY}

sociaux, politiques, culturels ou économiques auxquels ils sont dits correspondre dans les politiques publiques. Notre conclusion ouvre sur la nécessité d'une plus grande considération de la pratique elle-même et des facteurs qui influencent celle-ci.

\section{Repères historiques}

Selon Fedorov (2007), le mouvement liant éducation et médias, né en Europe, se concentrait au départ sur la presse et le cinéma, et sa genèse, qui prit place entre les années 1920 et 1940, fut principalement française ${ }^{5}$. La vitalité du cinéma français du début du $20^{\mathrm{e}}$ siècle aurait favorisé le développement de clubs de cinéma, la tenue de conférences nationales et l'instauration d'" offices régionaux du cinéma éducateur " dédiés à l'éducation, à l'enseignement et à la propagande par le cinéma (Laborderie, 2012). Des initiatives ciblant spécifiquement les jeunes, tels que CinéJeunes, furent développées avec pour objectif le développement de la pensée critique, des goûts et des habiletés créatives des enfants (Chevallier, 1980). De même, la fondation du British Film Institute (BFI) en 1933 encouragea également le développement d'enseignements se concentrant sur le cinéma au Royaume-Uni (ceux-ci gagnèrent en maturité quelques décennies plus tard). Également développée au Royaume-Uni au cours des années 1950, la Society for Education in Film and Television $(\mathrm{SEFT})^{6}$ contribua à établir la base des études médiatiques britanniques sur lesquelles reposent désormais plusieurs des approches contemporaines de l'éducation aux médias (Bolas, 2009). Le terme screen education, conçu et employé par la SEFT, s'imposa également dans le monde anglo-saxon (et particulièrement au Canada) comme un concept clé de l'éducation aux médias, celui-ci impliquant une lecture critique des images fixes et en mouvement (Moore, 1969). Des démarches préliminaires d'éducation aux médias prirent également place au

\footnotetext{
${ }^{5}$ Laramée (1998) soutient toutefois que les débuts de l'éducation aux médias sont britanniques.

${ }^{6}$ La SEFT avait préalablement pour nom Society of Film Teachers.
} 
Articuler les dimensions constitutives de l'éducation aux médias

sein de l'Union des républiques socialistes soviétiques (URSS) ${ }^{7}$ au cours des années 1920. Au Canada, les travaux de Marshall McLuhan inspirèrent les précurseurs en éducation aux médias et contribuèrent à l'institutionnalisation de la communication comme discipline académique (Landry et Letellier, 2016). Les premiers regroupements "d'éducateurs " aux médias canadiens prirent place à la fin des années 1960 et se consolidèrent au cours des années 1980 (Andersen, Duncan, et Pungente, 1999; Landry, Basque, et Agbobli, 2015). Cette décennie vit également l'introduction progressive de l'éducation aux médias dans les curriculums scolaires sur la scène internationale.

Les années 1970-1980 virent l'éclosion de deux phénomènes. En premier lieu, sur la lancée de l'éducation cinématographique et, dans une moindre mesure, de l'éducation à la presse et à la télévision, des chercheurs, des praticiens et des intellectuels de nombreux pays - parmi lesquels la Finlande, la Norvège, la Suède, la Suisse, l'Allemagne, la Belgique, le Canada et l'Australie (Piette, 1996) - développèrent des études théoriques sur l'éducation aux médias et donnèrent le coup d'envoi à une série de pratiques éducatives. Celles-ci se sont par la suite développées et parfois se sont institutionnalisées dans des systèmes scolaires. II s'agit donc de l'amorce d'une certaine formalisation de l'éducation aux médias qui s'étendit progressivement au cours des décennies suivantes.

En second lieu, de premières démarches concertées apparurent à l'échelle internationale. L'UNESCO s'impliqua dans l'éducation aux médias au cours des années 1970 et entreprit de jouer depuis un rôle fédérateur, de recherche et de transmission des connaissances (Landry et Roussel, 2018). Le Conseil de l'Europe s'intéressa également à la question (Masterman et Conseil de l'Europe, 1988). Ce fut le début des premiers travaux internationaux en matière d'éducation aux médias.

7 Celles-ci se concentrèrent essentiellement sur la presse et le cinéma et étaient orientées autour de la propagande communiste. Elles furent néanmoins rapidement interrompues par le gouvernement avant de reprendre vers la fin des années 1950 et de s'intégrer davantage dans les milieux scolaires (Fedorov, 2007). 


\section{Normand LANDRY}

Les années 1990-2000 virent une certaine consolidation de l'éducation aux médias sur la scène internationale, tant sur le plan du développement des politiques publiques, du développement et de la structuration d'organismes s'y consacrant, et de la constitution du champ de recherche et de pratique (Hoechsmann et Poyntz, 2012). Cette consolidation fut néanmoins chamboulée par deux phénomènes: des transformations médiatiques majeures, associées au développement et à la diffusion accélérés du numérique; l'affirmation de nouveaux besoins, de nouvelles préoccupations touchant au développement de compétences et de savoirs sur les médias (Alvermann et Hagood, 2000 ; Lin et al., 2013; Livingstone, 2003). Nous reviendrons sur ces éléments dans les sections qui suivent. Notons pour l'instant qu'ils sont associés à un accroissement marqué de l'intérêt pour l'éducation aux médias, se traduisant par une croissance importante en recherche et par le développement des programmes de formation qui s'y consacrent.

\section{Baliser le champ de l'éducation aux médias}

La première et peut-être la plus significative difficulté à laquelle est confronté le néophyte en " éducation aux médias " consiste à en définir et à en baliser les contours.

Cet obstacle est d'abord le produit d'un champ ayant pour spécificité d'opérer une convergence disciplinaire. D'une part, l'éducation aux médias emprunte aux sciences de l'information et de la communication les outils conceptuels et théoriques qu'elle déploie, notamment dans ses perspectives critiques (Landry et Basque, 2015). Elle s'appuie plus spécifiquement sur les études culturelles et médiatiques. Elle s'inspire également de la sociologie et des sciences politiques et fait des emprunts fréquents aux sciences cognitives et à la psychologie (Buckingham et SeftonGreen, 1994 ; Hammer et Kellner, 2009 ; Kellner et Share, 2005 ; Potter, 2013b).

D'autre part, elle tire des sciences de l'éducation, plus spécifiquement du courant de la pédagogie critique duquel elle s'inspire largement, un corpus théorique, une orientation et des 
Articuler les dimensions constitutives de l'éducation aux médias

méthodes centrées sur le développement des capacités autonomes des apprenants (Masterman, 1985; Share, 2009a). Elle s'éloigne des méthodes pédagogiques magistrales courantes, jugées inefficaces et inadaptées, pour se concentrer sur des démarches exigeant de l'apprenant qu'il entreprenne un processus introspectif, créatif et collaboratif orienté notamment autour de l'analyse et de la déconstruction de "textes " médiatiques, ainsi que la production médiatique (Bragg, 2002 ; Hobbs, 2016 ; Landry et Basque, 2015 ; Share, 2009b).

L'originalité de l'éducation aux médias, et conséquemment sa pertinence sociale et académique, réside ainsi tout à la fois dans la synthèse théorique qu'elle effectue et la pédagogie qu'elle emploie afin de rejoindre un public composé prioritairement d'enfants, d'adolescents et de jeunes adultes (Martens, 2010). II s'agit d'un champ fondamentalement normatif, dont les finalités sont orientées par des projets éducatifs qui intègrent des valeurs et des objectifs sociaux et politiques. L'éducation aux médias s'aborde donc en tant que praxis, c'est-à-dire en tant que pratique transformative opérant un dialogue constant entre la pratique et la théorie, entre la réflexion et l'action (Kellner et Share, 2007).

\subsection{Fractures et renouvellement}

Au sein de la communauté scientifique, des débats perdurent sur les assises théoriques et conceptuelles de l'éducation aux médias, sur ses finalités et ses priorités et sur les meilleures pratiques (Hobbs, 2011 ; Martens, 2010 ; Potter, 2013b ; Von Feilitzen et Carlsson, 2003). II s'agit, pour reprendre les propos de Ciurel (2016, p. 13), d'un « champ fracturé ».

Ces discussions sont séculaires. Elles perdurent et s'intensifient toutefois alors que se généralise la diffusion de médias numériques au sein de nos sociétés, que se transforment les industries médiatiques, les habitudes de production et de consommation médiatiques, qu'émergent ou que s'affirment des usages et des pratiques novatrices des technologies médiatiques numériques, et que s'imposent, dans l'espace public, de nouveaux enjeux (Eshet-Alkalai, 2004; Livingstone, 2003; Scheibe et Rogow, 2012). 


\section{Normand LANDRY}

Ces transformations posent comme défi le renouvellement de l'éducation aux médias. Les fondations théoriques, conceptuelles et pédagogiques de l'éducation aux médias ont été développées au $20^{\mathrm{e}}$ siècle, alors que la communication médiatique de masse constituait le mode dominant de production et de diffusion de l'information et que s'imposaient dans l'espace public des préoccupations et des besoins lui étant associés (Landry et Basque, 2015). Elles sont désormais confrontées à un environnement médiatique convergent, interactif, individualisé, démocratisé et fonctionnant en réseau.

Quatre axes peuvent être mobilisés pour présenter la nécessité de renouvellement.

Un premier axe, orienté autour de l'économie politique des médias, fait état de l'effondrement des modèles économiques traditionnels de production et de diffusion de la culture et de l'information, de l'émergence de nouveaux modèles économiques reposant sur l'accumulation, la capitalisation et la vente de données personnelles, et d'une convergence industrielle autour d'entreprises de haute technologie se faisant tout à la fois producteurs de dispositifs et diffuseurs de contenus, de plateformes et de services. Les concepts de concentration, de convergence, de propriété, d'industrie culturelle, d'idéologie et d'hégémonie - incontournables au sein des approches critiques en éducation aux médias - se réactualisent dans une économie politique de la production et de la diffusion médiatique radicalement transformée (Kellner et Share, 2005 ; Potter, 2013a).

Un second axe concerne la technologie médiatique elle-même, orientée autour de communications interactives et personnalisées, une capacité pour l'utilisateur de rejoindre de vastes audiences ou des groupes ciblés, une démocratisation marquée de la production de la diffusion de contenus, une ouverture à la programmation (et à la reprogrammation) des dispositifs mobiles, réseautés et connectés. Les compétences nécessaires à leur maîtrise évoluent en parallèle et trouvent, à mesure qu'ils se déploient et se généralisent dans les différents secteurs de la vie en société, une importance accrue (Jenkins, 2009 ; Lessig, 2008). À cet égard, les compétences nécessaires à la maîtrise de l'information et des 
Articuler les dimensions constitutives de l'éducation aux médias

dispositifs numériques occupent une importance considérable en éducation aux médias (Lee et al., 2013 ; UNESCO et al., 2013). Ces compétences sont directement en lien avec la notion d'inégalités numériques; elles sont réputées réduire les écarts existants dans les usages et les pratiques qui mobilisent les dispositifs numériques (Van Deursen et Van Dijk, 2011).

Ces innovations technologiques nourrissent à leur tour de nouveaux usages et de nouvelles pratiques médiatiques - notre troisième axe -, ceux-ci étant orientés autour d'une capacité à l'appropriation (autorisée ou illégale) de contenus, à la production et à la diffusion, de la personnalisation et la reconfiguration des dispositifs eux-mêmes (Hands, 2011; Landry, Pilote, et Brunelle, 2017). Ces usages et ces pratiques sont constitutifs de modes d'expression culturelle et politique à part entière, au sein de sociétés fortement médiatisées.

Finalement, le quatrième axe renvoie à la combinaison des facteurs industriels, technologiques et sociaux susmentionnés. Cet alliage contribue à l'affirmation de nouveaux enjeux et à la réactualisation de préoccupations séculaires associées aux médias. Il s'en suit conséquemment un impératif de réactualisation des compétences recherchées au sein d'environnements médiatiques évoluant rapidement (Buckingham, 2008), et s'y ajoute le développement de compétences et de savoirs spécifiquement orientés autour des enjeux du numérique, tels que la surveillance en ligne et la cyberintimidation (Livingstone et Haddon, 2009 ; Shariff, 2008).

Les changements associés au numérique et la nécessité qui en découle de renouveler l'éducation aux médias se traduisent par la multiplication de textes proposant des cadres intégrateurs, des clarifications ou des innovations conceptuelles (Addison et Meyers, 2013 ; Stordy, 2015).

Les transformations médiatiques et technologiques des dernières décennies ont été accompagnées d'une multiplication de discours mettant en exergue un impératif sociétal de développement de compétences associées à la maîtrise de l'information et des dispositifs médiatiques. Les écrits anglosaxons et, dans une moindre mesure, la production scientifique 


\section{Normand LANDRY}

francophone regroupent couramment ces compétences à l'intérieur de catégories conceptuelles associées à la notion de littératie médiatique (Livingstone, 2004).

Cette notion évoque une capacité à lire, à comprendre, à décoder, à produire et à analyser des textes, désormais " médiatiques », qu'elle contextualise dans le cadre des besoins des individus et des collectivités évoluant au sein des sociétés (Landry et Letellier, 2016). Elle demeure l'objet de débats récurrents et ne fait pas l'objet d'une définition consensuelle (Potter, 2013b). La notion de littératie médiatique (media literacy) s'impose néanmoins dans les écrits de langue anglaise comme un concept central à partir duquel il convient d'investir le champ de l'éducation aux médias.

\subsection{La question des littératies}

Le développement récent d'une myriade de "nouvelles littératies " associées à l'information, aux technologies et aux contenus numériques ajoute également à la confusion et au bruit conceptuel qui caractérise l'état des écrits (Landry et Basque, 2015).

Deux phénomènes se constatent depuis une quinzaine d'années : la multiplication de "littératies » plus spécifiques : ICT Literacy (Markauskaite, 2006), Internet/Web Literacy (Bawden, 2001 ; Fahser-Herro et Steinkuehler, 2010), Cyberliteracy (Gurak, 2008), Computer Literacy (Horton, 1983) et le développement de " méta-littératies » plus englobantes, telles que Multiliteracies (Provenzo et al., 2011), Metaliteracies (Mackey et Jacobson, 2011), new literacies (Greenhow et Robelia, 2009), media and information literacy (UNESCO et al., 2013) (Tableau 1) . Les écrits consultés sont consensuels quant à la nécessité d'organiser la multiplicité et l'hétérogénéité des concepts et des approches employés par les différents auteurs (Addison et Meyers, 2013; Aharony, 2010 ; Bawden, 2001 ; Erstad et Amdam, 2013 ; Lee et al., 2013 ; Stordy, 2015). Ce travail reste à accomplir. 
Articuler les dimensions constitutives de l'éducation aux médias

Tableau 1. Littératies

\begin{tabular}{|l|l|}
\hline Concepts & Chercheurs \\
\hline Media and information literacy & (Unesco et al., 2013) \\
\hline Information literacy & (Ercegovac, 2001) \\
\hline Media literacies & (Hoechsmann et Poyntz, 2012) \\
\hline Digital literacy & (Buckingham, 2008) \\
\hline Digital media literacies & (Buckingham, 2007) \\
\hline Digital literacies & (Jones et Hafner, 2012) \\
\hline Infomedia literacy & (Lee, 1999) \\
\hline Multiliteracies & (Provenzo et al., 2011) \\
\hline Virtual literacies & (Merchant et al., 2012) \\
\hline Technological literacy & (Dakers, 2006) \\
\hline Multimedia literacy & (Hobbs, 2006) \\
\hline Multimodal media literacy & (Lim, Nekmat, et Nahar, 2012) \\
\hline $\begin{array}{l}\text { Computer literacy (hardware } \\
\text { literacy, software literacy, } \\
\text { application literacy }\end{array}$ & (Horton, 2008) \\
\hline ICT literacy & \\
\hline Internet/web literacy & (Markauskaite, 2006) \\
\hline Cyberliteracy & $\begin{array}{l}\text { (Bawden, 2001 ; Fahser-Herro } \\
\text { et Steinkuehler, 2010) }\end{array}$ \\
\hline Transliteracy & (Gurak, 2008) \\
\hline Metaliteracies & (Frau-Meigs, 2012; Ipri, 2010) \\
\hline New literacies & (Jacobson et Mackey, 2013) \\
\hline Multiliteracies & (Greenhow et Robelia, 2009) \\
\hline & (Selber, 2004) \\
\hline
\end{tabular}




\section{Normand LANDRY}

Cette liste n'est pas exhaustive; Stordy (2015) recense plus d'une quarantaine de concepts. Pour Hobbs (2010) :

Each term is associated with a particular body of scholarship, practice and intellectual heritage, with some ideas stretching back to the middle of the $20^{\text {th }}$ century and other ideas emerging in the past couple of years. These terms reflect both the disciplinary backgrounds of the stakeholders and the wide scope of the knowledge and skills involved (p. 17).

Cette prolifération de littératies traduit une vitalité et une diversité appréciables au sein des écrits savants. Elle pose néanmoins un ensemble de problèmes.

En premier lieu, plusieurs auteurs soulignent le climat de confusion qu'entraîne la multiplication de littératies (Addison et Meyers, 2013 ; Stordy, 2015). Cette situation n'est pas nouvelle et a été constatée au tournant du siècle dernier (Bawden, 2001). La multidisciplinarité du champ s'avère être une particularité importante contribuant à l'éclatement des définitions et des concepts (Aharony, 2010). De plus, les liens conceptuels entre les différentes notions et approches disciplinaires sont peu explicites dans les écrits scientifiques. Des débats récurrents continuent à prendre place quant à la hiérarchisation et l'articulation des concepts (Lee et al., 2013; Martens, 2010) et de nouvelles littératies sont développées sur une base récurrente (Erstad et Amdam, 2013). Qui plus est, les processus de mise en application de plusieurs de ces concepts dans le cadre de pratiques pédagogiques sont peu détaillés ; l'évaluation du développement des compétences recherchées est conséquemment lacunaire. En somme, l'opérationnalisation de plusieurs de ces concepts demeure problématique (Hartai, 2014).

Dans ce contexte, il apparaît pertinent de définir le champ de l'éducation aux médias non pas à partir de ces ensembles de compétences et de savoirs, ceux-ci se modulant en fonction des préférences, des biais, des priorités et du positionnement de

\footnotetext{
${ }^{8}$ Ainsi, la question du développement de l'esprit critique (centrale en éducation aux médias) pose comme exigence l'application d'une définition opératoire du terme qui intègre des savoirs et des savoir-faire identifiés que l'on mesure, évalue et développe par l'enseignements et les pratiques sélectionnés.
} 
Articuler les dimensions constitutives de l'éducation aux médias

chacun, mais à partir de la pratique, de la praxis, elle-même. C'est pourquoi nous proposons la définition suivante de l'éducation aux médias, qui ne présume pas de ses finalités :

L' "éducation aux médias " réfère à l'ensemble des processus pédagogiques et didactiques de développement des compétences et des savoirs spécifiques sur les médias, relativement à des enjeux et des objectifs sociaux, politiques, économiques ou culturels (Landry et Roussel, 2018, p. 6).

La notion de médias est évoquée au sein des écrits de langue anglaise d'une manière analogue au langage courant, celle-ci évoquant, selon les cas, "des institutions qui produisent et diffusent des contenus, des acteurs socioéconomiques qui offrent des services (plateformes de réseaux sociaux, applications mobiles, moteurs de recherche, etc.), ou des technologies, lorsqu'elles sont associées à la production, à l'accès ou à la distribution de contenus " (Landry, Brunelle et Pilote, 2017, p. 2). $\mathrm{Si}$ elle engendre une certaine confusion, cette plasticité sémantique favorise également l'intégration d'un vaste éventail de thèmes et d'objets.

\section{L'articulation}

L'articulation constitue un élément clé pour évaluer la pertinence de la contribution des initiatives déployées en éducation aux médias. Elle pose une question essentielle: quels savoirs transmettre, par quels processus pédagogiques et enseignements, pour développer quelles compétences, afin d'atteindre quels objectifs, pour quels publics? L'articulation touche conséquemment quatre éléments: les savoirs théoriques, qui regroupent tout à la fois une posture épistémologique, un appareil conceptuel et un ensemble de propositions sur les médias, leurs rôles, leur fonctionnement, leurs fonctions et impacts; des pratiques pédagogiques, par lesquelles ces savoirs sont transmis et assimilés; des compétences que l'on cherche à développer des savoir-agir, des savoir-faire, des savoir-être notamment - en lien avec les "médias"; des objectifs sociaux, économiques, politiques ou culturels préidentifiés, parfois institutionnalisés et 


\section{Normand LANDRY}

codifiés par des politiques et des lois. Chacun de ces éléments constitue une voie d'entrée privilégiée au champ mobilisé par les experts et les praticiens. L'articulation renvoie donc au degré de cohérence entre ces éléments. Nous les aborderons dans les sous-sections suivantes.

\subsection{Savoirs}

Les savoirs véhiculés en éducation peuvent être structurés selon qu'ils s'intéressent à des thèmes, à des concepts ou à des approches.

\subsubsection{Thèmes}

Martens (2010) met en lumière quatre thèmes ou facettes des médias qu'il affirme structurer le champ: les industries médiatiques (soulevant ici la question de l'économie politique de la communication médiatisée); les messages médiatiques (considérés ici comme des "textes " pouvant être décortiqués, analysés et reconstruits) ; les publics (sujets à « influence », mais disposant également d'une capacité à interpréter, réinterpréter et agir sur les messages); les effets des médias, sur les plans individuel et social. Buckingham (2003) privilégie quant à lui l'étude de la production médiatique, le langage (médiatique), la représentation et les publics.

Tout en considérant le caractère éclectique de l'éducation aux médias, Potter (2013) identifie un ensemble de thèmes communs au sein des écrits savants : les effets des médias sur les individus ; l'influence sociale et politique des médias de masse ; la question de cette influence et de la passivité relative des audiences; la dualité entre la protection et la capacitation face aux médias; l'intervention pédagogique comme levier incontournable du développement des compétences et des savoirs recherchés.

En retraçant les racines de la littératie médiatique, Hobbs (2016) met en exergue les éléments suivants comme étant constitutifs du champ de l'éducation aux médias: la prise de conscience de la forme, du contenu et du contexte médiatiques, la nature socialement construite de la représentation et de l'interprétation des contenus médiatiques, la dialectique historique, suivant Potter (2014), entre protection et capacitation de l'enfance; les arts et 
Articuler les dimensions constitutives de l'éducation aux médias

l'activisme médiatiques; et, finalement, les apprentissages supportés par les dispositifs, les formats et les contenus médiatiques ou encore dirigés sur ceux-ci.

Le développement et la diffusion massive de technologies médiatiques numériques se sont accompagnés de thèmes qui leur sont consacrés. Livingstone (2004) et Buckingham (2007) proposent tous deux des cadres qui réarticulent des thèmes " traditionnels " en éducation aux médias dans un contexte numérique (l'accès aux médias, l'analyse des contenus, l'évaluation critique, la création; le langage médiatique, la production médiatique, les audiences et l' « écriture " (la production de « textes » médiatiques). Les thèmes qui émergent en parallèle au numérique concernent notamment les questions de l'exercice de la citoyenneté (Mihailidis et Thevenin, 2013), des droits numériques des enfants (Livingstone et O'neill, 2014), des liens entre compétences, fractures et inégalités numériques (Müller, Sancho, et Hernández, 2009), des risques numériques, des comportements, des usages et des pratiques numériques (FrauMeigs, 2011).

\subsubsection{Concepts}

Une lignée établie d'acteurs et d'auteurs s'inscrivant dans une tradition nord-américaine et britannique structure le champ de l'éducation aux médias autour d'un certain nombre de principes fréquemment appelés "concepts »- qui en balisent la programmation. Ces concepts (Tableau 2) découlent directement ou indirectement des travaux de Len Masterman (Jolls et Wilson, 2014 ; Landry et al., 2015 ; Pungente, Duncan, et Andersen, 2005). Ils constituent des affirmations touchant à des catégories thématiques distinctes et qui posent à l'apprenant des questions spécifiques, le conviant, dans une démarche introspective, à l'expérimentation et à la résolution de problèmes. 
Tableau 2. Concepts

\begin{tabular}{|l|l|}
\hline Concepts & Catégories thématiques \\
\hline $\begin{array}{l}\text { Media messages are } \\
\text { constructed. }\end{array}$ & $\begin{array}{l}\text { Examen des processus de } \\
\text { production médiatique, selon les } \\
\text { genres et les formats. }\end{array}$ \\
\hline $\begin{array}{l}\text { Media messages are } \\
\text { produced within economic, } \\
\text { social, political, historical and } \\
\text { aesthetic contexts. }\end{array}$ & $\begin{array}{l}\text { Analyse et déduction des } \\
\text { intentions et des motivations des } \\
\text { producteurs et diffuseurs de } \\
\text { contenus médiatiques ; analyse } \\
\text { des modèles économiques de la } \\
\text { production et de la diffusion de } \\
\text { contenus médiatiques; examen } \\
\text { des impacts sociaux et politiques } \\
\text { de la circulation de ces contenus. }\end{array}$ \\
\hline $\begin{array}{l}\text { The interpretative meaning- } \\
\text { making processes involved in } \\
\text { message reception consist of } \\
\text { an interaction between the } \\
\text { reader, the text and the } \\
\text { culture. }\end{array}$ & $\begin{array}{l}\text { Mise en lumière des processus } \\
\text { de négociation de sens entre } \\
\text { audiences, textes et auteurs; } \\
\text { effets sociaux des médias ; } \\
\text { cultures et sous-cultures } \\
\text { médiatiques. }\end{array}$ \\
\hline $\begin{array}{l}\text { Media have unique } \\
\text { "languages," characteristics } \\
\text { which typify various forms, } \\
\text { genres and symbol systems of } \\
\text { communication. }\end{array}$ & $\begin{array}{l}\text { Étude des formats et des genres } \\
\text { médiatiques; études des effets, } \\
\text { des impacts, des influences de la } \\
\text { rhétorique visuelle, sonore et } \\
\text { textuelle utilisé ; études } \\
\text { sémiologiques de contenus } \\
\text { médiatiques. }\end{array}$ \\
\hline $\begin{array}{l}\text { Media representations play a } \\
\text { role in people's understanding } \\
\text { of social reality. } \\
\text { (Aufderheide, 1993, cité dans } \\
\text { Potter, 2004, p. 259.) }\end{array}$ & $\begin{array}{l}\text { Étude des rapports entretenus } \\
\text { entre les publics, les messages } \\
\text { et les producteurs dans la } \\
\text { négociation du « réel », de } \\
\text { l'authentique; études } \\
\text { journalistiques. }\end{array}$ \\
\hline
\end{tabular}


Articuler les dimensions constitutives de l'éducation aux médias

Différentes variations de ces concepts sont mobilisées par les chercheurs et de nombreux organismes d'éducation aux médias. Ceux-ci ont structuré le champ de l'éducation aux médias sur la scène internationale :

The frameworks developed by the Association for Media Literacy in Canada and the British Film Institute in England (which are closely related) have been very influential internationally, even in very different cultural contexts. Most countries that have an explicit framework use some variant of these, while others appear to have adopted one or other of them wholesale. (Domaille et Buckingham, 2001, p. 15.)

Ces « concepts " accomplissent trois fonctions: introduire un certain nombre d'affirmations présentées comme des assises de l'éducation aux médias; aborder des catégories thématiques particulières; favoriser l'introspection, la réflexion critique et le dialogue sur les questions discutées. Pour les promoteurs d'une structuration des savoirs basés sur ces concepts clés, ceux-ci jettent les bases d'un "cadre systématique, cohérent, reproductible, mesurable et évolutif à l'échelle mondiale et donc, intemporel » (Jolls et Wilson, 2014, p. 68).

\subsubsection{Approches}

Les savoirs véhiculés en éducation aux médias se structurent également en fonction d'approches. Celles-ci témoignent des grandes orientations normatives conférées aux démarches pédagogiques privilégiées. Ces orientations sont, à leur tour, tributaires de conceptions spécifiques de ce que sont et font les médias et, conséquemment, des savoirs et des compétences devant être développés. Quatre approches sont recensées: protectionniste, artistique, synthétique et critique (Hartai, 2014 ; Kellner et Share, 2007 ; Piette, 1996).

Développée dès les années 1930, l'approche protectionniste positionne l'éducation aux médias de manière défensive, dans une optique de protection contre les effets néfastes des médias.

Ce fut dès le départ un mouvement défensif et paternaliste dont l'objectif était d'introduire les formes courantes de médias dans la salle de classe dans le seul but de les dénigrer parce que commerciales, manipulatrices et sans 


\section{Normand LANDRY}

aucune originalité. [...] L'éducation aux médias fut ainsi, à ses débuts, une éducation contre les médias (Masterman, 1994, p. 22).

La démarche favorise la critique de productions médiatiques massifiées, inscrites dans le cadre d'une industrialisation de la culture et de la reproduction de l'idéologie dominante. L'approche protectionniste perdure toujours: elle se trouve néanmoins réactualisée autour de discours mettant en exergue les risques des environnements médiatiques pour des populations considérées comme vulnérables (enfants, adolescents, minorités), notamment en ce qui a trait à la violence, à la sexualité et aux rapports de genres, aux comportements antisociaux, à l'influence culturelle et psychologique sur les comportements, les attitudes et gles estes (Erstad et Amdam, 2013 ; Frau-Meigs, 2011).

L'approche artistique trouve ses racines au cours des années 1960 (Loicq, 2011). Selon Hoechsmann et Poyntz (2012), il s'agit d'une période de croissance importante de l'éducation aux médias ayant conduit à des expérimentations en classe au Royaume-Uni, au Canada, en France aux États-Unis et en Australie. L'approche artistique fut orientée à ses débuts vers l'analyse de productions cinématographiques désormais traitées comme des "œuvres" pouvant faire l'objet d'une critique esthétique et culturelle. Nourrie par le développement des études cinématographiques survenu dans les années 1950-1960 en Europe et en Amérique du Nord, cette approche fut d'abord qualifiée de " sélective " puisqu'elle se concentrait essentiellement sur le cinéma (et, dans une moindre mesure, sur la télévision aux États-Unis), négligeant d'autres médias. Fedorov (2007) résume ainsi les objectifs de l'approche esthétique employée par les éducateurs britanniques au cours des années 1960 :

- accroître auprès des élèves la compréhension et le plaisir de la télévision et du cinéma ;

- promouvoir leur apprentissage de la société humaine et de leur individualité ;

- outiller les élèves pour une autodéfense face à la manipulation commerciale ; 
Articuler les dimensions constitutives de l'éducation aux médias

- encourager l'expression personnelle, non seulement par les formes traditionnelles (prise de parole, écriture, dessin), mais aussi par le langage de l'écran (faire des films).

En vertu de cette approche, le sens critique, selon Masterman (1994), est utilisé « non pas contre les médias, mais contre leur contenu » (p. 8) et vise la critique culturelle. L'approche artistique se concentre essentiellement sur les textes médiatiques, au détriment d'une perspective plus sociologique sur l'analyse des contextes de leur production, de leur diffusion et de leur réception. L'approche artistique est désormais réactualisée par les études s'insérant à la créativité et à l'innovation numériques, associées notamment aux productions culturelles basées sur le recyclage culturel, la collaboration, l'expérimentation de formes artistiques convergentes et multimédias (Buckingham, 2008).

L'approche synthétique, associée par Piette (1996) à une phase historique de "consolidation" de l'éducation aux médias, se constitua progressivement autour des années 1970-1980, essentiellement autour des travaux de Masterman. Inspirée par les travaux de Roland Barthes, de Louis Althusser, d'Antonio Gramsci et de Raymond Williams, l'éducation aux médias devait dès lors considérer les médias comme des sites de "représentation » du monde social, où des « interprétations opposées de la réalité se font concurrence » (Raboy, 1996, p. 14) et où s'expriment des conflits portant sur la définition, la présentation et la légitimation des codes culturels et des narratifs sociaux. Cette approche, qui s'impose toujours, s'oppose à une dévalorisation anticipée de la culture populaire et des médias, mais reconnaît et apprécie plutôt leurs contributions, tout en cherchant à comprendre leurs actions ainsi qu'à les situer à l'intérieur de leurs contextes sociaux, politiques, économiques et culturels. Elle intègre tant la production que l'analyse médiatique.

Finalement, l'approche critique, tout en intégrant des aspects des approches susmentionnées, se concentre sur les questions associées au pouvoir, à la domination, à la représentation (des genres, des groupes éthiques, des minorités sexuelles) (Kellner et Share, 2005; Share, 2009b). Elle est fondamentalement ancrée dans une dimension de capacitation, cherchant à développer chez 


\section{Normand LANDRY}

les apprenants les ressources nécessaires à la pensée et au jugement critique, mais également - et simultanément - à la transformation et au changement social (Landry et al., 2017). Elle est ainsi orientée autour du développement d'une capacité d'action. L'approche critique trouve ses outils conceptuels et pédagogiques dans les études culturelles et postcoloniales, au sein des écrits sur les médias alternatifs, communautaires et radicaux, de même que les études de mouvements sociaux.

\subsubsection{Perspectives}

Qu'ils soient structurés sous le couvert de thèmes, de concepts ou d'approches, les savoirs véhiculés en éducation aux médias jettent sur les médias un regard mettant en lumière quatre perspectives complémentaires: culturelle, sociopolitique, langagière et de production.

Ces perspectives constituent autant d'angles par lesquels sont élaborées des activités pédagogiques. Elles contribuent, en outre, à l'intégration de l'éducation aux médias dans différentes disciplines scolaires: la perspective culturelle favorise ainsi l'intégration de préoccupations associées à l'éducation aux médias dans des cours de littérature, d'art, d'histoire de l'art et de langues; la perspective sociopolitique propose une intégration similaire dans les cours de sociologie, de sciences politiques, de communication et de philosophie; la perspective langagière s'impose dans le domaine de la formation aux arts visuels, la littérature, la communication et les sciences politiques; la perspective de production est pertinente dans le cadre de formations en journalisme, en communication ou en techniques appliquées des médias.

\subsection{Pratiques et politiques}

Un mouvement international sur l'éducation aux médias est porté depuis plus de quarante ans par des éducateurs, des chercheurs, des parents, des universitaires, des autorités du milieu de l'éducation et des fonctionnaires. Ce mouvement s'applique tout à la fois à structurer le champ de l'éducation aux médias, à en étendre les pratiques et à l'inscrire dans le cadre d'enseignements formels prodigués en milieu scolaire. 
Articuler les dimensions constitutives de l'éducation aux médias

Le développement et l'analyse de politiques publiques en éducation aux médias ont débuté au cours des années 1980, alors que se structuraient les premiers programmes disposant d'une reconnaissance institutionnelle et politique formelle (Masterman et Conseil de l'Europe, 1988 ; Piette, 1996 ; UNESCO, 1982).

La situation diffère toujours grandement d'un pays, voire d'une région ou d'une localité à l'autre, certains programmes d'éducation aux médias étant le fruit d'initiatives communautaires, d'autres se positionnant comme des démarches volontaires d'enseignants passionnés et certains étant formellement intégrés (à des degrés divers), dans des curriculums scolaires (Hartai, 2014 ; Oea, 2016). Ces efforts sont soutenus par divers organismes communautaires, scolaires et administratifs qui s'investissent dans le domaine de l'éducation.

La structuration et la progression du champ au cours des dernières décennies se sont illustrées par la multiplication des déclarations, des chartes et des recommandations internationales (Landry et Basque, 2015). L'UNESCO occupe une place croissante sur la scène internationale sur la question ${ }^{9}$. L'organisme mobilise désormais la notion d'éducation aux médias et à l'information (media and information literacy) comme concept composite intégrateur regroupant les différentes littératies numériques, informationnelles et médiatiques. Cette intégration traduit une tendance croissante sur la scène internationale - et notamment française - à intégrer au sein du champ de l'éducation aux médias les contributions des sciences de l'information (Lee et al., 2013). Elle positionne les compétences informationnelles (savoir chercher, analyser, évaluer et produire l'information) comme étant centrales dans un environnement médiatique désormais largement numérique.

\footnotetext{
${ }^{9}$ L'Unesco développe tout à la fois des guides à l'intention des éducateurs, des guides de développement et d'évaluation des politiques nationales en éducation aux médias et à l'information et des guides à l'intention des radiodiffuseurs. Elle soutient le développement d'un réseau international de partenaires internationaux appelé Global Alliance for Partnerships on Media and Information Literacy (GAPMIL) et le développement d'un réseau international d'universités participant à la recherche et à l'éducation.
} 


\section{Normand LANDRY}

Les organismes qui investissent actuellement le champ des politiques publiques en éducation aux médias regroupent désormais des institutions internationales (Commission européenne ${ }^{10}, \mathrm{UNESCO}^{11}$ ), des agences nationales (Conseil supérieur de l'éducation aux médias ${ }^{12}$, Office of communications $\left[\mathrm{Ofcom}^{13}\right]$ ), les centres d'expertise et de diffusion $\left(\mathrm{CLEMI}{ }^{14}\right)$, des organisations non gouvernementales (National Association for Media Literacy Education ${ }^{15}$, HabiloMédias, ${ }^{16}$ Association for Media Literacy ${ }^{17}$ ) et des regroupements professionnels (Fédération canadienne des enseignantes et enseignants ${ }^{18}$ ). Les experts confirment que la société civile occupe désormais un "rôle de premier plan » dans le développement et la mise en œuvre d'activités en éducation aux médias (Observatoire européen de l'audiovisuel, 2016).

Le développement des politiques concerne toutefois, au premier chef, les états nationaux. Sans être exhaustif, le Tableau 3 illustre quelques-uns des principaux secteurs concernés.

\footnotetext{
${ }^{10}$ Voir http://ec.europa.eu/culture/policy/audiovisual-policies/literacy_en.

${ }^{11}$ Voir http://www.unesco.org/new/en/communication-and-information/capacity-buildingtools/media-and-information-literacy/.

${ }^{12}$ Voir http://www.csem.be.

${ }^{13}$ Voir https://www.ofcom.org.uk/research-and-data/media-literacy.

${ }^{14}$ Voir http://www.clemi.fr/fr.html.

${ }^{15}$ Voir https://namle.net.

${ }^{16}$ Voir http://habilomedias.ca.

${ }^{17}$ Voir http://www.aml.ca.

${ }^{18}$ Voir https://www.ctf-fce.ca/en/Pages/Issues/Media-Literacy.aspx.
} 
Tableau 3. Politiques

\begin{tabular}{|l|l|}
\hline Secteurs & Politiques et réglementations \\
\hline $\begin{array}{l}\text { Réglementation des } \\
\text { médias }\end{array}$ & $\begin{array}{l}\text { Réglementation de la publicité ; conduite } \\
\text { d'activités de recherche sur les effets et } \\
\text { influences des médias, les pratiques et les } \\
\text { usages médiatiques. }\end{array}$ \\
\hline $\begin{array}{l}\text { Politiques } \\
\text { éducatives }\end{array}$ & $\begin{array}{l}\text { Adoption de curriculums scolaires } \\
\text { intégrant l'éducation aux médias; } \\
\text { formation des enseignants; intégration de } \\
\text { la technologie à l'école; évaluation des } \\
\text { apprentissages sur les médias } \\
\text { (notamment numériques) ou mobilisant les } \\
\text { médias numériques; soutien aux } \\
\text { bibliothèques scolaires et publiques. }\end{array}$ \\
\hline $\begin{array}{l}\text { Politiques } \\
\text { communautaires }\end{array}$ & $\begin{array}{l}\text { Programmes de soutien aux médias } \\
\text { communautaires, étudiants, alternatifs, } \\
\text { autochtones. }\end{array}$ \\
\hline Politiques familiales & $\begin{array}{l}\text { Politiques d'accès des foyers aux } \\
\text { technologies et aux réseaux de } \\
\text { communication numérique ; aide au } \\
\text { développement des compétences } \\
\text { médiatiques des parents. }\end{array}$ \\
\hline Criminalité/droit civil & $\begin{array}{l}\text { Adoption de législations sur la } \\
\text { cyberintimidation, le harcèlement, la } \\
\text { diffamation, la production et la distribution } \\
\text { de matériel sous droit d'auteur, de } \\
\text { pornographie juvénile ou non } \\
\text { consensuelle; formation des corps } \\
\text { policiers et sensibilisation du grand public. }\end{array}$ \\
\hline Politiques sur des & $\begin{array}{l}\text { Adoption de programmes et de législation } \\
\text { visant la sensibilisation au droit à la vie } \\
\text { privée, à l'exercice de la citoyenneté dans } \\
\text { des environnements numériques, etc. }\end{array}$ \\
\hline
\end{tabular}


Un corpus se constitue autour de l'analyse comparative de politiques publiques en éducation aux médias (Buckingham et Domaille, 2002 ; Frau-Meigs et Torrent, 2009 ; Frau-Meigs, Velez, et Flores Michel, 2017 ; UNESCO et al., 2013). La plupart des pays européens et nord-américains ont développé de telles politiques (Oea, 2016).

Malgré ces développements, la recherche sur les politiques publiques en éducation aux médias demeure peu développée et lacunaire sur trois aspects particuliers : les modalités d'inscription de l'éducation aux médias dans les curriculums scolaires, l'influence de cette inscription sur les pratiques pédagogiques déployées en classe et l'efficacité de ces pratiques, compte tenu des orientations curriculaires et des objectifs de développement de compétences et de savoirs chez les élèves.

L'inscription de l'éducation aux médias au sein des curriculums scolaires permet d'en généraliser les pratiques, dans une optique de développement de savoirs et de compétences priorisés et de réponse aux enjeux et aux préoccupations soulevées par la médiatisation des sociétés. Ainsi considérés, les curriculums scolaires sont au cœur des politiques éducatives, puisqu'ils établissent tout à la fois les priorités éducatives, définissent les orientations disciplinaires, attribuent les responsabilités aux différents acteurs scolaires et définissent les mesures d'évaluation (Landry et Roussel, 2018).

Les travaux qui procèdent à l'analyse critique des curriculums scolaires en matière d'éducation aux médias demeurent rares ${ }^{19}$ (Buckingham et Domaille, 2002 ; Kirwan et al., 2003 ; Tanriverdi et Apak, 2010). À notre connaissance, les travaux réalisés sous la direction de László Hartai (2014) constituent la démarche la plus aboutie en la matière. Ceux-ci mettent en lumière non seulement les inscriptions formelles de l'éducation aux médias dans les curriculums scolaires des pays européens, mais aussi les facteurs

\footnotetext{
${ }^{19}$ Le projet European Media Literacy Education Study (EMEDUS) constitue certainement l'une des initiatives qui attaque le plus directement de front les lacunes dans les connaissances en matière de politiques éducatives. Voir le site à cette adresse: http://www.gabinetecomunicacionyeducacion.com/en/research/european-media-literacyeducation-study-emedus.
} 
qui influencent directement le développement des apprentissages à l'école. À cet égard, la formation du personnel enseignant, les conditions de pratiques, l'évaluation formelle des contenus associés à l'éducation aux médias apparaissent fondamentales.

En affirmant que "l'étude de l'éducation aux médias effectuée à l'école constitue le véritable site inexploré » du champ (Hartai, 2014, p. 43), Hartai et ses collaborateurs mettent en lumière une faiblesse majeure dans les écrits: ceux-ci se préoccupent insuffisamment de l'articulation entre savoirs, pratiques, compétences et politiques publiques, notamment scolaires. Les connaissances sur les contenus curriculaires en éducation aux médias demeurent parcellaires et incomplètes; cette situation proscrit une analyse critique de la qualité des contenus et une évaluation de leur arrimage avec les savoirs scientifiques et les pratiques déployées par les professionnels sur le terrain.

Les travaux de Hartai ainsi que nos propres travaux (Landry et Roussel, 2018) conduisent à deux conclusions. D'une part, dans le contexte où l'éducation aux médias se conçoit en tant que praxis, il est pour le moins étonnant, près de quatre décennies après sa consolidation et son entrée dans les politiques éducatives, que peu de travaux s'intéressent à la pratique elle-même, lorsque déployée dans un cadre scolaire, et aux facteurs qui influencent cette pratique. D'autre part, le renforcement de l'articulation en éducation aux médias pose comme exigence le développement des savoirs sur les curriculums scolaires et sur les pratiques enseignantes. Nous proposons quelques axes (Tableau 4), touchant tout à la fois aux contenus des programmes et aux pratiques enseignantes devant les traduire en activités et en apprentissages. 
Tableau 4. Contenus circulaires et pratiques en milieu scolaire

\begin{tabular}{|c|c|c|c|c|c|c|}
\hline \multicolumn{7}{|c|}{ Contenus } \\
\hline \multicolumn{2}{|c|}{ Intégration des savoirs scientifiques } & \multicolumn{3}{|c|}{$\begin{array}{l}\text { Logique intégratrice des contenus à l'intérieur } \\
\text { des curriculums }\end{array}$} & \multicolumn{2}{|c|}{$\begin{array}{c}\text { Prescriptions curriculaires en lien avec les } \\
\text { médias pour différentes catégories } \\
\text { d'acteurs }\end{array}$} \\
\hline \multicolumn{2}{|c|}{$\begin{array}{l}\text { Dans quelle mesure et de quelle manière } \\
\text { les contenus curriculaires intègrent-ils les } \\
\text { savoirs scientifiques? }\end{array}$} & \multicolumn{3}{|c|}{$\begin{array}{c}\text { Comment est intégrée l'éducation aux } \\
\text { médias dans le curriculum ? Où se trouvent } \\
\text { ses principaux ancrages? Quels contenus } \\
\text { sont évalués? }\end{array}$} & \multicolumn{2}{|c|}{$\begin{array}{l}\text { Que demande-t-on aux acteurs } \\
\text { (enseignants, écoles, personnel scolaire, } \\
\text { etc.) de faire ou d'être en mesure de faire } \\
\text { en lien avec les médias? }\end{array}$} \\
\hline \multicolumn{7}{|c|}{ Pratique enseignante } \\
\hline $\begin{array}{l}\text { Connaissance et } \\
\text { compréhension des } \\
\text { contenus curriculaires }\end{array}$ & \multicolumn{2}{|c|}{$\begin{array}{l}\text { Processus de traduction } \\
\text { des contenus curriculaires } \\
\text { en pratiques pédagogiques }\end{array}$} & $\begin{array}{l}\text { Soutien au } \\
\text { développement et au } \\
\text { déploiement des } \\
\text { pratiques pédagogiques }\end{array}$ & \multicolumn{2}{|c|}{$\begin{array}{l}\text { Ressources mises à la } \\
\text { disposition des } \\
\text { enseignants }\end{array}$} & $\begin{array}{l}\text { Formation dont } \\
\text { disposent les } \\
\text { enseignants }\end{array}$ \\
\hline $\begin{array}{l}\text { Quelles connaissance } \\
\text { et compréhension des } \\
\text { contenus en éducation } \\
\text { aux médias possèdent } \\
\text { les enseignants? }\end{array}$ & \multicolumn{2}{|c|}{$\begin{array}{c}\text { Quelles sont les pratiques } \\
\text { pédagogiques déployées } \\
\text { en milieu scolaire en } \\
\text { éducation aux médias? } \\
\text { Comment et dans quelle } \\
\text { mesure les enseignants } \\
\text { traduisent-ils les contenus } \\
\text { curriculaires en activités et } \\
\text { pratiques pédagogiques? }\end{array}$} & $\begin{array}{l}\text { De quel degré de soutien } \\
\text { et de reconnaissance } \\
\text { professionnelle les } \\
\text { enseignants qui } \\
\text { développement des } \\
\text { activités en éducation aux } \\
\text { médias bénéficient-ils? }\end{array}$ & \multicolumn{2}{|c|}{$\begin{array}{c}\text { De quelles ressources } \\
\text { (techniques, matérielles, } \\
\text { humaines, temporelles) } \\
\text { disposent les enseignants } \\
\text { pour développer les } \\
\text { apprentissages et le } \\
\text { développement de } \\
\text { compétences sur les } \\
\text { médias? }\end{array}$} & $\begin{array}{c}\text { Dans quelle mesure la } \\
\text { formation initiale et } \\
\text { contenue des } \\
\text { enseignants intègre-t- } \\
\text { elle des composantes } \\
\text { en éducation aux } \\
\text { médias? }\end{array}$ \\
\hline
\end{tabular}


Ces thèmes et ces questions n'épuisent pas les secteurs d'investigation que l'on peut retrouver en milieu scolaire. Ils mettent toutefois en lumière deux éléments. Premièrement, le développement des savoirs en éducation aux médias exige que l'on s'intéresse aux conditions, aux milieux et aux acteurs qui en structurent la pratique. Deuxièmement, la consolidation du champ de l'éducation aux médias passe nécessairement par le renforcement de l'articulation entre savoirs, pratiques pédagogiques, compétences et objectifs (ici intégrés aux curriculums). Les disjonctions constatées entre les axes de l'éducation aux médias - cette autonomie partielle de chacun de ces éléments, leur arrimage approximatif les uns aux autres, et la récurrence de zones d'ombre dans les savoirs qui s'y rapportent constituent une limite à dépasser.

\section{Conclusion}

L'analyse des écrits de langue anglaise permet de tirer quelques conclusions générales sur le champ de l'éducation aux médias.

L'une de ses plus appréciables contributions réside dans la synthèse théorique qu'elle articule. Cette synthèse fait converger, par une posture résolument transdisciplinaire, des outils conceptuels permettant de jeter un éclairage sur les phénomènes médiatiques et rendant ces derniers plus intelligibles. Cette synthèse est nécessairement pédagogique, puisqu'elle se préoccupe tant des savoirs qu'elle met en forme que des manières de les transmettre. Cette attention particulière accordée à une mise en forme pédagogique d'un corpus de connaissances constitue un trait caractéristique du champ de l'éducation aux médias. Sur ce point précis, il se distingue des études culturelles et médiatiques.

L'éducation aux médias demeure néanmoins confrontée à une double difficulté. D'une part, elle est tiraillée entre une tendance au morcellement qui priorise le développement de savoirs et de compétences spécifiques, associés à des technologies, des enjeux ou des thèmes particuliers, et une prétention à recouvrir l'entièreté 
des phonèmes associés à la médiatisation des sociétés et aux rapports établis entre enfants, jeunes et médias. Ce tiraillement nourrit une inflation conceptuelle dont nous avons discuté. Celle-ci rend, à son tour, plus difficile l'identification des fondements, des priorités et des éléments constitutifs de l'éducation aux médias.

D'autre part, l'intérêt marqué pour l'identification, la classification et l'organisation pédagogique des savoirs et des compétences recherchés semble s'être jusqu'à récemment accompagné d'un relatif désintérêt vis-à-vis des contextes à l'intérieur desquels se déploient les activités d'enseignement. Cette lacune trouve désormais réponse dans les travaux inédits de chercheurs européens. Les connaissances sur les politiques en éducation aux médias, sur leur mise en œuvre et leur impact, sur les facteurs qui influent les pratiques restent également à être développées, particulièrement en Amérique du Nord.

La consolidation du champ semble conséquemment nécessiter une articulation plus affirmée des dimensions constitutives de l'éducation aux médias. Une telle démarche suppose non seulement une valorisation accrue des aspects négligés par la recherche, mais également une meilleure mise en relation de la théorie, de la pratique et des politiques. Cela oblige à revenir à des questions de base: quels contenus, thèmes et savoirs sont privilégiés en éducation aux médias? Par quels processus pédagogiques ceux-ci sont-ils transmis, et avec quelle efficience ? Afin de développer quelles compétences? En lien et soutenues par quelles politiques? Celles-ci étant mises en œuvre par quels acteurs, dans quels contextes et avec quels impacts ?

Ainsi formulée, la notion d'articulation suppose que l'on considère ces interrogations dans leur totalité. L'identification des disjonctions, des écarts entre les éléments qu'elles soulèvent - par exemple, la mise en lumière d'incohérences entre des pratiques pédagogiques particulières et les compétences qu'elles doivent concourir à développer - ouvre de riches agendas de recherche. Plus fondamentalement, une telle démarche permet de situer l'éducation aux médias en tant que pratique se déployant dans des conditions qui la contraignent ou la libèrent. La richesse du champ tient à sa particularité : son objet est une pratique multiforme qui 
se module en fonction des priorités, des objectifs, des contextes, des acteurs et des milieux où elle s'inscrit. II en découle une exigence d'intégration dans la recherche des facteurs internes et externes qui en influencent le développement et le déploiement.

\section{Références}

ADDISON C. et E. MEYERS, 2013, « Perspectives on information literacy: A framework for conceptual understanding ", Information Research: An International Electronic Journal, vol. $18, \mathrm{n}^{\circ} 3$.

AHARONY N., 2010, "Information literacy in the professional literature: An exploratory analysis », Aslib Proceedings, vol. 62, $\mathrm{n}^{\circ} 3$, pp. 261-282.

ALVERMANN D. E. et M. C. HAGOOD, 2000, «Critical media literacy: Research, theory, and practice in "new times" », The Journal of Educational Research, vol. 93, n 3, pp. 193-205.

ANDERSEN N., B. DUNCAN et J. PUNGENTE, 1999, "Media education in Canada - The second spring ", dans C. VON FEILITZEN et U. CARISSON (dir.), Children and media: Image, education, participation. Children and media violence. Yearbook from the UNESCO international clearinghouse on children and violence on the screen, Göteborg, UNESCO, Nordicom, pp. 139-162.

BAWDEN D., 2001, «Information and digital literacies: A review of concepts ", Journal of Documentation, vol. $57, \mathrm{n}^{\circ} 2$, pp. 218259.

BOLAS T., 2009, Screen education from film appreciation to media studies. Bristol, Intellect.

BRAGG S., 2002, "Wrestling in woolly gloves: Not just being critically media literate », Journal of Popular Film and Television, vol. 30, $\mathrm{n}^{\circ} 1$, pp. 41-51.

BUCKINGHAM D., 2007, «Digital media literacies: Rethinking media education in the age of the internet », Research in Comparative and International Education, vol. 2, $\mathrm{n}^{\circ}$ 1, p. 43-55. 
BUCKINGHAM D., 2008, « Defining digital literacy: What do young people need to know about digital media? ", dans C. LANKSHEAR et M. KNOBEL (dir.), Digital literacies: Concepts, policies and practices, Oxford, Peter Lang, pp. 73-91.

BUCKINGHAM D. et K. DOMAILLE, 2002, Where are we going and how can we get there? General findings from the UNESCO youth media education survey 2001, University of Southampton, Centre for Language in Education, UNESCO.

BUCKINGHAM D. et J. SEFTON-GREEN, 1994, Cultural studies goes to school: Reading and teaching popular media, Londres, Taylor \& Francis.

CHEVALLIER J. (dir.), 1980, Cine-club et action éducative. Paris, CNDP.

CIUREL D., 2016, "Media literacy. Concepts, approaches and competencies ", Professional communication and translation studies, vol. 9, pp. 13-20.

DAKERS J. (dir.), 2006, Defining technological literacy: Towards an epistemological framework. New York, Palgrave Macmillan.

DOMAILLE, K. et D. BUCKINGHAM, 2001, Youth media education survey, Paris, UNESCO.

ERCEGOVAC Z., 2001, Information literacy: Search strategies, tools \& resources for high school students, Worthington, Linworth.

ERSTAD O. et S. AMDAM, 2013, "From protection to public participation: A review of research literature on media literacy », Javnost, vol. 20, $\mathrm{n}^{\circ} 2$, pp. 83-98.

ESHET-ALKALAI Y., 2004, "Digital literacy: A conceptual framework for survival skills in the digital era », Journal of Educational Multimedia and Hypermedia, vol. 13, pp. 93-106.

FAHSER-HERRO D. et C. STEINKUEHLER, 2010, «Web 2.0 literacy and secondary teacher education $»$, Journal of Computing in Teacher Education, vol. 26, $\mathrm{n}^{\circ}$ 2, pp. 55-62.

FEDOROV A., 2007, Media education: A historical perspective, $<$ http://www.mediagram.ru/netcat_files/108/110/h_b5cfa7db9d2 
Articuler les dimensions constitutives de l'éducation aux médias

16d26989e2b1ec147ba5f>, dernière consultation le 24 avril 2017.

FRAU-MEIGS D., 2011, Socialisation des jeunes et éducation aux médias: du bon usage des contenus et comportements à risque, Toulouse, Éditions Érès.

FRAU-MEIGS D., 2012, "Transliteracy as the new research horizon for media and information literacy », Medijske Studije, vol. $3, n^{\circ} 6$, pp. 14-27.

FRAU-MEIGS D. et J. TORRENT (dir.), 2009, Mapping media education policies in the world: Visions, programmes and challenges, New York, UN-Alliance of Civilizations.

FRAU-MEIGS D., I. VELEZ et J. FLORES MICHEL (dir.), 2017, Public policies in media and information literacy in Europe. Cross-country comparisons, New York, Routledge.

GREENHOW C. et B. ROBELIA, 2009, «Old communication, new literacies: Social network sites as social learning resources", Journal of Computer-Mediated Communication, vol. 14, $\mathrm{n}^{\circ} 4$, pp. 1130-1161.

GURAK L. J., 2008, Cyberliteracy: Navigating the internet with awareness, New Haven, Yale University Press.

HAMMER R. et D. KELLNER (dir.), 2009, Media/cultural studies: Critical approaches, New York, Peter Lang.

HANDS J., 2011, @ is for activism: Dissent, resistance and rebellion in a digital culture, Londres/New York, Pluto.

HARTAI L., 2014, Report on formal media education in Europe 2014, Hungarian Institute for Education Research and Development.

HOBBS R., 2006, «Multiple visions of multimedia literacy: Emerging areas of synthesis ", International handbook of literacy and technology, vol. 2, pp. 15-28.

HOBBS R., 2010, Digital and media literacy: A plan of action, Washington, The Aspen Institute. 
HOBBS R., 2011, "The state of media literacy: A response to potter », Journal of Broadcasting \& Electronic Media, vol. 55, $\mathrm{n}^{\circ}$ 3, pp. 419-430.

HOBBS R., 2016, Exploring the roots of digital and media literacy through personal narrative, Philadelphie, Temple University Press.

HOECHSMANN M. et S. R. POYNTZ, 2012, Media literacies: A critical introduction, Malden/Hichester, Wiley-Blackwell.

HORTON F. W. J., 2008, Understanding information literacy: A primer, Paris, UNESCO.

HORTON F. W. J., 1983, "Information literacy vs. Computer literacy ", American Society for Information Science Bulletin, vol. $9, n^{\circ} 4$, pp. 14-16.

IPRI T., 2010, «Introducing transliteracy what does it mean to academic libraries? », College \& Research Libraries News, vol. 71, $\mathrm{n}^{\circ} 10$, pp. 532-567.

JACOBSON T.E. et T.P. MACKEY, 2013, «Proposing a metaliteracy model to redefine information literacy ", Communications in information literacy, vol. $7, \mathrm{n}^{\circ}$ 2, pp. 84-91.

JENKINS H., 2009, Confronting the challenges of participatory culture: Media education for the $21^{\text {st }}$ century, Cambridge, The MIT Press.

JOLLS T. et C. WILSON, 2014, « The core concepts: Fundamental to media literacy yesterday, today and tomorrow », Journal of Media Literacy Education, vol. 6, $\mathrm{n}^{\circ}$ 2, pp. 68-78.

JONES R. H. et C.A. HAFNER, 2012, Understanding digital literacies: A practical introduction. Abingdon/New York Routledge.

KELLNER D. et J. SHARE, 2005, " Toward critical media literacy: Core concepts, debates, organizations, and policy ", Discourse: Studies in the Cultural Politics of Education, vol.26, $\mathrm{n}^{\circ} 3$, pp. 369-386.

KELLNER D. et J. SHARE, 2007, "Critical media literacy is not an option », Learning Inquiry, vol. 1, n 1, pp. 59-69. 
Articuler les dimensions constitutives de l'éducation aux médias

KIRWAN T. et al., 2003, Mapping media literacy: Media education 11-16 years in the united kingdom, Londres, BFI.

LABORDERIE P., 2012, "Les offices du cinéma scolaire et éducateur à l'épreuve des publics ", Conserveries mémorielles, vol. 12 , <http://cm.revues.org/1230>, dernière consultation le 24 avril 2017.

LANDRY N. et J. BASQUE, 2015, «L'éducation aux médias: Contributions, pratiques et perspectives de recherche en sciences de la communication ", Communiquer. Revue de communication sociale et publique, $\mathrm{n}^{\circ} 15, \mathrm{pp} .47-63$.

LANDRY N., J. BASQUE et C. AGBOBLI, 2015, «Éducation aux médias au Canada: état des savoirs et perspective de recherche en communication », dans A. KIYINDOU (dir.), Éducation aux médias. Nouveaux enjeux, rôles et statuts des acteurs, Paris, L'Harmattan.

LANDRY N. et A.-S. LETELLIER (dir.), 2016, Éducation aux médias : fondations, perspectives et enjeux, Montréal, Presses de l'Université de Montréal.

LANDRY N., A.-M. PILOTE et A.-M. BRUNELLE, 2017, "L'éducation aux médias en tant que pratique militante : luttes et résistances au sein des espaces médiatiques et de gouvernance ", dans M. BONENFANT, F. DUMAIS et G. TRÉPANIER-JOBIN (dir.), Les pratiques transformatrices de l'espace socionumérique: appropriation, résistance et expérimentation, p. 119-139.

LANDRY N. et C. ROUSSEL, 2018, "Élèves, éducation aux médias et citoyenneté : analyse du programme de formation de l'école québécoise ", Lien social et politiques, vol. 80, 38 p.

LARAMÉE A., 1998, L'éducation critique aux médias, Sainte-Foy, Télé-université.

LEE A. et al., 2013, Conceptual relationship of information literacy and media literacy in knowledge societies, Paris, UNESCO. 
LEE A. Y. L., 1999, «Infomedia literacy: An educational basic for young people in the new information age », Information, Communication \& Society, vol. 2, $\mathrm{n}^{\circ}$ 2, pp. 134-155.

LEE A. Y. L., 2010, "Media education: Definitions, approaches and development around the globe ", New Horizons in Education, vol. 58, $\mathrm{n}^{\circ}$ 3, pp. 1-13.

LESSIG L., 2008, Remix: Making art and commerce thrive in the hybrid economy, New York, Penguin Press.

LIM S. S., E. NEKMAT et S. N. NAHAR, 2012, " The implications of multimodality for media literacy », Multimodal Studies: Exploring Issues and Domains, vol. 2, p. 169-183.

LIN T.-B. et al., 2013, "Understanding new media literacy: An explorative theoretical framework ", Educational Technology \& Society, vol. 16, n 4, pp. 160-170.

LIVINGSTONE S., 2003, The changing nature and uses of media literacy,

$<$ http://eprints.Ise.ac.uk/13476/1/The changing nature and us es_of media_literacy.pdf>, dernière consultation le 24 avril 2017.

LIVINGSTONE S., 2004, "What is media literacy? ", Intermedia, vol. 32, n 3, pp. 18-20.

LIVINGSTONE S. et B. O'NEILL, 2014, "Children's rights online: Challenges, dilemmas and emerging directions ", dans S. VAN DER HOF, B. VAN DEN BERG et B. SCHERMER (dir.), Minding minors wandering the web: Regulating online child safety, La Haye, Springer, pp. 19-38.

LIVINGSTONE S. M. et L. HADDON (dir.), 2009, Kids online: Opportunities and risks for children, Bristol/Portland, Policy Press.

LOICQ M., 2011, Médias et interculturalite : l'éducation aux médias dans une perspective comparative internationale, Thèse de doctorat, Université Sorbonne Nouvelle - Paris III/Université Laval. 
MACKEY T.P. et T. E. JACOBSON, 2011, « Reframing information literacy as a metaliteracy ", College and Research Libraries, vol. 72, $\mathrm{n}^{\circ}$ 1, pp. 62-78.

MARKAUSKAITE L., 2006, "Towards an integrated analytical framework of information and communications technology literacy: From intended to implemented and achieved dimensions ", Information Research: An International Electronic Journal, vol. 11, n 3, p. 252-277.

MARTENS H., 2010, "Evaluating media literacy education: Concepts, theories and future directions ", Journal of Media Literacy Education, vol. 2, n 1, pp.1-22.

MASTERMAN L., 1985, Teaching the media, Londres/New York, Routledge.

MASTERMAN L., 1994, L'éducation aux médias dans l'Europe des années 90 un guide pour les enseignants, Strasbourg, Strasbourg Conseil de l'Europe.

MASTERMAN L. et CONSEIL DE L'EUROPE, 1988, Le développement de l'éducation aux médias dans l'Europe des années 80: en particulier dans le domaine de la télévision et des médias électroniques, Strasbourg, Conseil de la coopération culturelle, Conseil de l'Europe.

MERCHANT G. et al. (dir.), 2012, Virtual literacies: Interactive spaces for children and young people, New York, Routledge.

MIHAILIDIS P. et THEVENIN B., 2013, « Media literacy as a core competency for engaged citizenship in participatory democracy ", American Behavioral Scientist, vol. 57, $\mathrm{n}^{\circ} 11$, pp. 1611-1622.

MOORE G. J., 1969, "The case for screen education », dans F.K. STEWART et J. NUTTALL (dir.), Screen education in canadian schools, Toronto, Canadian Education Association, pp. 6-26.

MÜLLER J., SANCHO J.M. et HERNÁNDEZ F., 2009, « New media literacy and the digital divide. Taking into account the socio-economic dimension of ict", dans L. W. H. TAN et 
R. SUBRAMANIAM (dir.), Handbook of research on new media literacy at the k-12 level: Issues and challenges, Hershey, Information Science Reference, pp. 72-88.

OEA, 2016, Mapping of media literacy practices and actions in eu28, Strasbourg, Conseil de l'Europe.

PIETTE J., 1996, Éducation aux médias et fonction critique. Paris /Montréal, L'Harmattan.

POTTER J. W., 2013a, Media literacy ( $7^{e}$ éd.), Santa Barbara, University of California.

POTTER J. W., 2013b, "Review of literature on media literacy », Sociology Compass, vol. 7, $n^{\circ}$ 6, pp. 417-435.

PROVENZO E. F. et al., 2011, Multiliteracies: Beyond text and the written word, Charlotte, Information Age Pub.

PUNGENTE J. J., B. DUNCAN et N. ANDERSEN, 2005, "The canadian experience: Leading the way ", Yearbook Nat Soc Study Education Yearbook of the National Society for the Study of Education, vol. 104, n 1, pp. 140-160.

RABOY M., 1996, Occasions ratées histoire de la politique canadienne de radiodiffusion. Montréal/Sainte-Foy, Liber/ Presses de l'Université Laval.

SCHEIBE C. et F. ROGOW, 2012, The teacher's guide to media literacy: Critical thinking in a multimedia world, Thousand Oaks, Corwin.

SELBER S., 2004, Multiliteracies for a digital age, Carbondale, SIU Press.

SHARE J., 2009a, Media literacy is elementary: Teaching youth to critically read and create media, New York, Peter Lang.

SHARE J., 2009b, "Young children and critical media literacy ", dans D. KELLNER et R. HAMMER (dir.), Media/cultural studies: Critical approaches, New York, Peter Lang Publishers, pp. 126151. 
Articuler les dimensions constitutives de l'éducation aux médias

SHARIFF S., 2008, Cyber-bullying: Issues and solutions for the school, the classroom and the home, Londres/New York, Routledge.

STORDY P., 2015, "Taxonomy of literacies », Journal of Documentation Journal of Documentation, vol. $71, \mathrm{n}^{\circ} 3$, pp. $456-$ 476.

TANRIVERDI B. et O. APAK, 2010, "Analysis of primary school curriculum of turkey, finland, and ireland in terms of media literacy education », Educational Sciences: Theory and Practice, vol. $10, n^{\circ} 2$, pp. 1187-1213.

UNESCO, 1982, Déclaration de grunwald sur l'éducation aux médias, <http://www.unesco.org/education/pdf/MEDIA_F.PDF> dernière consultation le 7 mars 2017.

UNESCO et al., 2013, Global media and information literacy (mil). Assessment framework: Country readiness and competencies, Paris, Unesco.

VAN DEURSEN A. et J. VAN DIJK, 2011, « Internet skills and the digital divide ", New Media and Society, vol. 13, n 6, pp. 893911.

VON FEILITZEN C. et U. CARLSSON (dir.), 2003, Promote or protect?: Perspectives on media literacy and media regulations, Göteborg, The International Clearinghouse on Children/Youth and Media. 